\section{FRI0628 DIGITAL TECHNOLOGIES TO PROMOTE SELF- MANAGEMENT IN MUSCULOSKELETAL HEALTH: A SYSTEMATIC REVIEW}

L. Sammut ${ }^{1}$, N. Arafin ${ }^{2}$, Y. Prior ${ }^{2} .{ }^{1}$ Rheumatology, University Hospital Southampton NHS Foundation Trust, Southampton, ${ }^{2}$ School of Health Sciences, University of Salford, Manchester, United Kingdom

Background: With the emergence of musculoskeletal conditions as a major threat to health status and one of the largest causes of health care expenditures, the role of self-management strategies has emerged. Digital media as a platform for self management interventions in musculoskeletal health is a new and evolving landscape in health care.

Objectives: To review the published evidence to support the self-management of rheumatic and musculoskeletal conditions through digital technologies. Methods: MEDLINE, EMBASE, PsycINFO, Global Health, The Cochrane Library (Cochrane Database of Systematic Reviews, Cochrane Central Register of Controlled Trials (CENTRAL), Cochrane Methodology Register), and Web of Science (science and social science citation index) were searched and the papers selected were screened by two independent reviewers. Methodological validity prior to inclusion was assessed using the appropriate critical appraisal tools developed by the Critical Appraisal Skills Programme (CASP).

Results: Database searches identified 5179 studies. Five randomised controlled trials assessing digital media in the self management of musculoskeletal diseases were selected. The participants in the studies were predominately females with a mean age of 50 . The mean number of participants in the studies was 140.8 (range 92-228). Two studies targeted self management strategies for patients diagnosed with rheumatoid arthritis [1, 2]. One study included fibromyalgia patients [3] and the two other studies included patients that reported arthritis pain [4] or general non cancer related pain [5]. A robust and meaningful comparison between the studies was infeasible because of heterogeneity between studies and small sample size in the studies. Positive effects of self-management were demonstrated in arthritis self-efficacy, pain, physical functioning, opioid misuse and overall quality of life.

Conclusions: All studies showed an improvement in a number of different selfmanagement outcomes. Digital media may be effective in the delivery of self-management programmes of patients with rheumatic and musculoskeletal conditions. Further research is necessary to increase the scope of these findings using rigorous study designs so we can harness the full potential of digital technologies as a medium for delivering self-management interventions.

\section{REFERENCES:}

[1] Shigaki CL, Smarr KL, Siva C, et al. RAHelp: an online intervention for individuals with rheumatoid arthritis. Arthritis care \& research 2013;65 (10):1573-1581.

[2] Van den Berg $\mathrm{MH}$, Ronday HK, Peeters AJ, et al. Using internet technology to deliver a home-based physical activity intervention for patients with rheumatoid arthritis: A randomized controlled trial. Arthritis Care \& Research 2006;55(6):935-945.

[3] Williams DA, Kuper D, Segar M, Mohan N, Sheth M, Clauw DJ. Internetenhanced management of fibromyalgia: a randomized controlled trial. PAIN $^{\circledR}$ 2010;151(3):694-702.

[4] Trudeau KJ, Pujol LA, DasMahapatra P, Wall R, Black RA, Zacharoff K. A randomized controlled trial of an online self-management program for adults with arthritis pain. Journal of behavioral medicine 2015;38(3):483496.

[5] Wilson M, Roll JM, Corbett C, Barbosa-Leiker C. Empowering patients with persistent pain using an internet-based self-management program. Pain Management Nursing 2015;16(4):503-514.

Disclosure of Interest: None declared

DOI: 10.1136/annrheumdis-2018-eular.7542

\section{FRI0629 PATIENT-TARGETED SMARTPHONE APPS FOR SYSTEMIC LUPUS ERYTHEMATOSUS: A SYSTEMATIC REVIEW AND ASSESSMENT OF FEATURES AND QUALITY}

M. Pourrahmat ${ }^{1,2, *}$, M. A. De Vera ${ }^{1,3} .{ }^{1}$ Arthritis Research Canada, Richmond, ${ }^{2}$ Faculty of Medicine, ${ }^{3}$ Faculty of Pharmaceutical Sciences, University of British Columbia, Vancouver, Canada

Background: Smartphone apps are readily accessible eHealth tools that may support the self-management of patients with systemic lupus erythematosus (SLE). However, knowledge on the availability and quality of apps targeted to this population is limited.
Objectives: To identify smartphone apps targeted to patients with SLE and to classify their functions and assess their quality using a reliable and objective scale.

Methods: We applied a systematic review framework to the search, screening, and assessment of apps. Android and iPhone apps were searched on the Google Play Store and the App Store, respectively in January 2018. Search terms included "lupus" or "SLE". Apps were included if they were: (1) smartphonebased; (2) compatible with the Android or the iOS operating systems; (3) in the English language; (4) targeted to people with SLE; and (5) available for download in either of the two app stores. Apps were excluded if they were: (1) targeting a condition other than SLE; (2) including only treatment algorithms; or (3) explicitly only for health care providers. App name, platform (Android, iPhone), developer current version, cost, and user star ratings and comments were extracted. The Mobile App Rating Scale was used to classify the apps.

Results: We identified 315 apps, including 249 from the Google Play Store and 66 from the App Store (figure 1). Of these, 32 met inclusion criteria (19 Android, 13 iPhone).

Of the 19 Android apps, 16 were free to download and the remaining cost $2.93-$ 5.65 CAD. The focus across apps were mainly to increase patient well-being. The theoretical background or strategies behind the apps varied, the most common ones included: providing information/education regarding SLE for the user (14 apps), providing advice/tips/strategies/skills training for SLE management (9 apps), and providing capabilities for monitoring/tracking of symptoms (6 apps). The user star ratings of the apps ranged from $1.5-5.0$ stars (out of 5.0 ) and the number of user ratings ranged from $1-288$. Common user critiques indicated the need for increased functionality in entering and tracking symptoms in apps that provided this feature.

Of the 13 iPhone apps, 10 were free to download and the remaining cost $2.79-$ 6.99 CAD. The focus across apps were mainly to increase patient well-being. The theoretical background or strategies behind the apps varied, the most common ones included: providing information/education regarding SLE for the user ( apps), providing capabilities for monitoring/tracking of symptoms (6 apps), and providing advice/tips/strategies/skills training for SLE management (5 apps). No user ratings or comments were available.

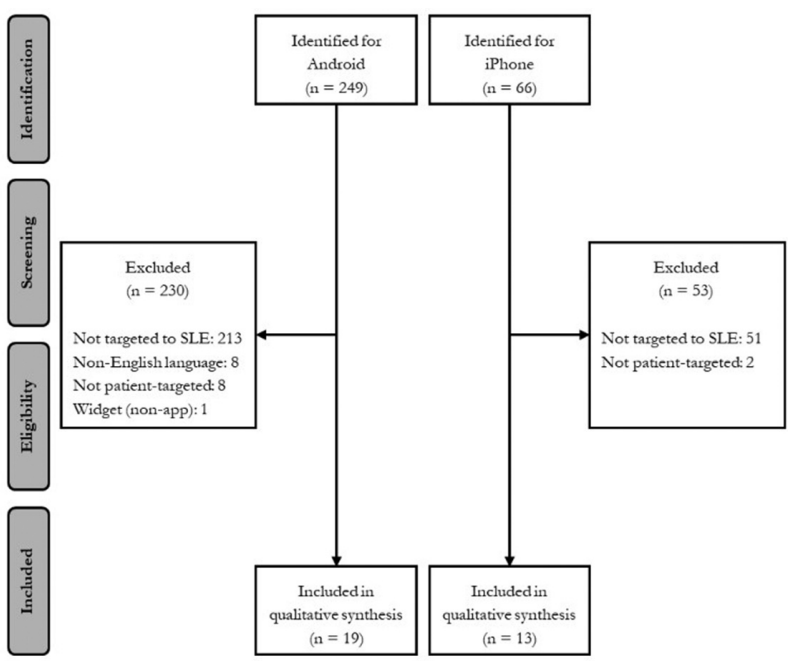

Conclusions: Applying a systematic review framework, we identified and classified 32 apps mainly focusing on increasing patient well-being in SLE. These findings have practical implications for helping patients identify potential eHealth tools to support self-management of SLE.

Disclosure of Interest: None declared

DOI: 10.1136/annrheumdis-2018-eular.7106

\section{FRI0630 CAN COMMUNITY PHARMACY SERVICES BE THE MISSING LINK IN ACHIEVING EARLY DIAGNOSIS OF PSORIATIC ARTHRITIS?}

P. Mehra ${ }^{1}$, M. K. Nisar ${ }^{2} .{ }^{1}$ Farley Hill pharmacy, ${ }^{2}$ Rheumatology, Luton \& Dunstable University Hospital, Luton, United Kingdom

Background: There is an urgent need to look at New Models of Care to improve quality of care for patients whilst providing the best value for money. Community Pharmacy has been an untapped resource which can assist in achieving the goals of managing long term conditions. Psoriasis is one such example affecting $1 \%$ to $3 \%$ of the population with upto $40 \%$ of these patients at risk of developing psoriatic 